\title{
Lymphomatous Involvement of Extranodal Site
}

National Cancer Institute

\section{Source}

National Cancer Institute. Lymphomatous Involvement of Extranodal Site. NCI

Thesaurus. Code C99567.

A pathologic and/or clinical finding indicating the spread of a lymphomatous process to an anatomic site other than lymph nodes. 